\title{
Diffuse alveolar haemorrhage and myocardial infarction: life-threatening effects from self-injected hyaluronic acid dermal filler
}

\author{
Amira Aishah Che Ani, Sakinah Mohd Akhir, Winnie Chiang Li-Xue, Nur Zurairah Shahidan, \\ Azman Abd Hamid \\ Department of Anaesthesiology and Critical Care, Hospital Tuanku Fauziah, Perlis, Ministry of Health, Malaysia
}

\begin{abstract}
Hyaluronic acid (HA) is a widely used dermal filler for soft tissue augmentation. We described a case of a 38 -year-old transwoman who presented with sudden onset of severe respiratory distress following self-injection of HA dermal filler. She developed multiple episodes of pulmonary haemorrhage, and her chest $\mathrm{X}$-ray showed diffuse ground-glass opacities consistent with diffuse alveolar haemorrhage (DAH). There were no relevant drugs or past medical histories. Anti-nuclear antibodies and rheumatoid factor were negative. Initially, the pulmonary haemorrhage
\end{abstract}

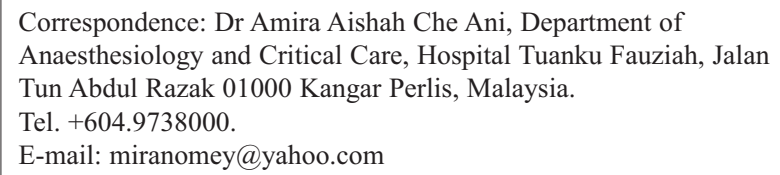

Key words: Hyaluronic acid; respiratory distress syndrome; hemorrhage; myocardial infarction; dermal fillers.

Contributions: AACA, planning, structuring, and initial writing of the report; WCL, NZS, responsible for transcribing medical notes; SMA, responsible for providing constructive criticism of the report; AACA, $\mathrm{AAH}$, responsible to finalise the draft prior to submission. All the authors have read and approved the final version of the manuscript and agreed to be accountable for all aspects of the work.

Conflict of interest: The authors declare that they have no competing interests, and all authors confirm accuracy.

Study Registration: The study has been registered with the National Medical Research Register (NMRR) of the Ministry of Health Malaysia (NMRR-21-258-58809).

Acknowledgements: The authors would like to thank the Director General of Health for his permission to publish the paper.

Received for publication: 12 March 2021.

Accepted for publication: 11 June 2021.

${ }^{\circ}$ Copyright: the Author(s), 2021

Licensee PAGEPress, Italy

Monaldi Archives for Chest Disease 2021; 91:1845

doi: 10.4081/monaldi.2021.1845

This article is distributed under the terms of the Creative Commons Attribution Noncommercial License (by-nc 4.0) which permits any noncommercial use, distribution, and reproduction in any medium, provided the original author(s) and source are credited. episodes and ventilation requirement improved with systemic steroid, however she subsequently developed acute myocardial infarction with progressive clinical deterioration leading to death. To the best of our knowledge, this is the first HA-related DAH with myocardial infarction reported with a fatal outcome. This case highlights the importance of awareness and the necessity of having a high suspicion of DAH in patients with history of illicit HA dermal filler use.

\section{Case Report}

A 38-year-old, previously well, transwoman presented to us with sudden onset of shortness of breath and non-productive cough. She denied systemic complaints and was a non-smoker. There was no travelling history nor contact with known COVID19 patients. Upon arrival, she appeared mildly tachypnoeic (respiratory rate of 24 breaths/min) with oxygen saturation of $89 \%$ under room air, requiring face mask $5 \mathrm{l} / \mathrm{min}$ to maintain good saturation. There was no documented fever and the haemodynamic parameters were stable, unsupported. Physical examinations revealed crepitations at right lower zone on auscultation and her chest X-ray showed bilateral diffuse ground-glass opacities (Figure 1).

Laboratory test revealed leucocytosis with neutrophilic predominance (total white cell count: $18.87 \times 10^{3} / \mu \mathrm{L}$ ); Neutrophil $89 \%$, Lymphocyte $6 \%$ ) and a slight increase in the C-reactive protein level $(22 \mathrm{mg} / \mathrm{L})$. Otherwise, other routine haematological parameters including renal and liver function panels were within normal limits.

Within 24 hours of admission, her oxygen requirement markedly increased. Her oxygen saturation ranged from 80 to $85 \%$ under high flow mask oxygen $15 \mathrm{l} / \mathrm{min}$. She was subsequently intubated and ventilated, requiring high ventilatory setting $\left(\mathrm{FiO}_{2}\right.$ of 1.0 and PEEP of 12) to maintain acceptable oxygenation. Immediately following intubation, copious amount of fresh blood was noted from the endotracheal tube suggestive of pulmonary haemorrhage. The coagulation profile and platelet count were within normal limits. Computed tomography imaging was not performed in view of her unstable condition for transport.

Leptospira serology, COVID-19 polymerase chain reaction (PCR) and D-dimer were negative. She did not improve despite given broad-spectrum antibiotic and continued to have episodes of pulmonary haemorrhage, resulting in a drop of $4 \mathrm{~g} / \mathrm{dl}$ of haemoglobin within hours and progressive decline in oxygenation status. Intravenous methylprednisolone $1 \mathrm{~g}$ daily were started and she was nursed in a prone position as per acute respiratory distress syndrome (ARDS) protocol. Her pulmonary haemorrhage episode 
improved, and subsequent improvement in oxygenation were temporarily observed.

Unfortunately, her inotropic support began to increase drastically within the next few hours. The 12-lead electrocardiogram (ECG) showed anterolateral ST-elevation with elevated TroponinI level of $4,000 \mathrm{pg} / \mathrm{ml}$. She continued to deteriorate clinically and eventually succumbed to death. Clinical post-mortem was not performed due to the absence of family members to provide consent. Microbiology testing, including test for acid fast bacilli and culture from blood and tracheal aspirate were negative. Infective screening for human immunodeficiency virus (HIV), hepatitis B, hepatitis C, and autoimmune screening, including anti-nuclear antibody (ANA) and rheumatoid factor (RF) were all insignificant. The antineutrophil cytoplasmic antibodies (ANCA) assay though indicated, was not performed due to unavailability of local resources.

Detailed social history revealed that she had lived as a transwoman for the past 11 years and took multiple injections for feminisation. She injected herself with different types of medications purchased online, including intravenous oestradiol, progesterone, vitamin $\mathrm{C}$, intravenous placenta, hyaluronidase and hyaluronic acid (HA) fillers. She had been taking these medications regularly with no previous complications. The HA fillers were routinely injected into the breasts and gluteal area with a total volume of $100 \mathrm{ml}$. Prior to her current admission, she had had history of injecting the HA dermal filler a few hours before developing respiratory symptoms.

\section{Discussion}

Self-injection dermal fillers are increasingly common social practice in the community. Hyaluronic acid (HA) has become the filler of choice as they are longer-lasting, less immunogenic and is hydrolysed by hyaluronidase [1]. Side effects are usually mild, transient and reversible. In this report, we highlighted a rare case of diffuse alveolar haemorrhage (DAH) and acute myocardial infarction following HA filler injection. We found only two cases of DAH following HA filler injection from our literature search $[2,3]$. These cases were diagnosed based on clinical presentation,

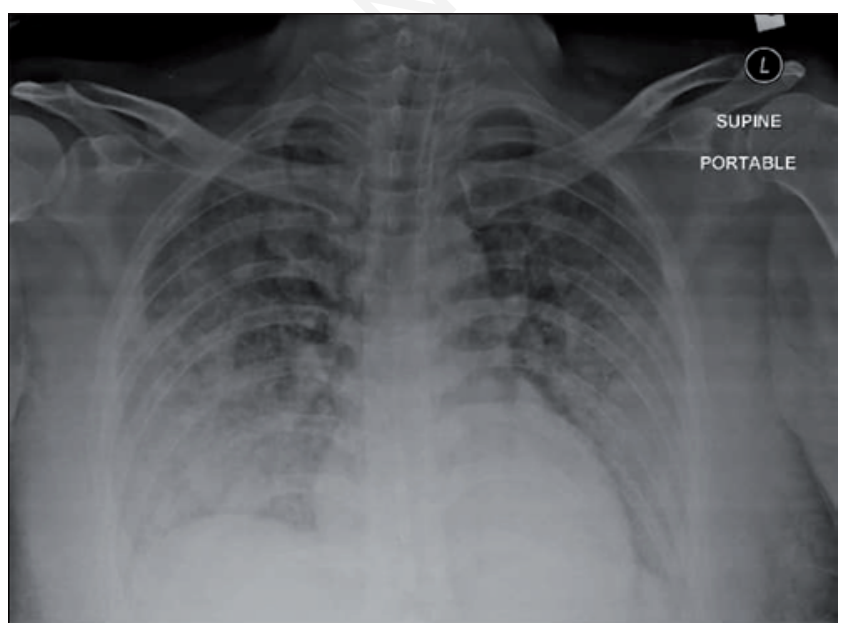

Figure 1. Chest $\mathrm{X}$-ray upon arrival showing diffuse ground-glass opacities. bronchoalveolar lavage (BAL) and computed tomography findings. Both patients did not require mechanical ventilation and responded well to steroid therapy with good clinical outcome. There were no reports of HA-related acute coronary syndrome as experienced by our patient.

DAH may present with haemoptysis, anaemia, and acute respiratory failure. Even though most DAH cases are associated with systemic autoimmune diseases, they may also result from coagulation disorders, drugs, inhaled toxins, or transplantation. Its diagnosis relies on clinical suspicion, combined with laboratory, radiologic, and pathologic findings [4]. Myocardial infarction is a clinical event diagnosed in the presence of raised cardiac enzymes, cardiacrelated symptomatology, and ECG changes suggestive of myocardial ischaemia. Various pathology underlies its occurrence, including coronary artery rupture, plaque erosion and vasospasm [5].

In our case, the patient's initial response to the steroid may suggest autoimmune vasculitic disease. While the ANCA assay could be a valuable diagnostic tool [6-8], its use is not clearly justified due to the lack of local resources, as the test will have to be outsourced, resulting in a delay in obtaining results, on top of the incurred additional expenses. Furthermore, the acute onset of respiratory distress experienced by our patient was not associated with other systemic symptoms to indicate infections or autoimmune disease, and that the clinical improvement seen with steroid therapy was only temporarily observed.

Even though we were not able to exclude pulmonary thromboembolism through computed tomography for pulmonary artery (CTPA), the low clinical probability from history and a negative Ddimer suggests the exclusion of thromboembolic event. Given the temporal association, the presence of pulmonary haemorrhage, and the exclusion of other possible causes, we postulated that the HA dermal filler's self-injection could be a rare cause of DAH.

We also speculated that the subsequent event of myocardial infarction could be a direct effect of HA. Although we cannot rule out type II myocardial infarction from hypoxaemia, myocardial infarction is uncommon in this age group, and this patient did not have the traditional risk factors associated with coronary artery disease. Therefore, the possibility of HA-related myocardial infarction cannot be ruled out. The development of deep-tissue scars fixed in arteries after frequent injections may predispose to high circulating HA volume, resulting in clinical toxicity [9]. Chronic opiate abuse is one of the aetiological factors for alveolar haemorrhage, however medical history and physical examination were not suggestive (i.e., absence of track marks). To the best of our knowledge, this is the first reported case of DAH and myocardial infarction following gluteal and breast HA filler injection with a fatal outcome.

The underlying mechanism of HA filler causing DAH and myocardial infarction is unknown. The exogenous HA were known to bind to specific HA receptor on endothelial cells. Furthermore, the lung endothelium expresses the highest level of HA receptors as compared to other cell lines, which may explain the early respiratory signs [10]. Therefore, an autopsy report would add considerable value to the case as the presence of certain histopathological features, such as myocardial degeneration with cardiac dilatation, may provide corroborative evidence for chemical-related fatal adverse reaction. However, a clinical post-mortem was not performed in our case due to the lack of consent.

HA may affect fibrin formation by decreasing the clotting time of fibrinogen by 3 to 10 folds [11], possessed heparin-like activity [12] and is associated with thrombocytopenia [13]. HA has also been implicated in the pathophysiology of lung injury and remodelling. Low molecular weight HA fragments may promote inflam- 
mation by stimulating the release of pro-inflammatory cytokines [14], potentially leading to a compromised alveolar-capillary barrier with eventual vascular leakage. HA may also act as a ligand to a toll-like receptor on coronary endothelial cell causing endothelial cells apoptosis. The denuded intimal surface of the coronary artery or plaque erosion may provoke the development of thrombosis, which is one of the pathogenesis of myocardial infarction [5].

However, the exact mechanism of DAH and myocardial infarction remains unclear in this case. However, we speculated that the high level of HA may trigger abnormal haemostasis and disrupt the alveolar-capillary integrity leading to DAH, causing plaque erosion in the coronary artery provoking thrombosis, hence acute myocardial infarction.

\section{Conclusions}

The case presented highlights the life-threatening effects of HA filler. Therefore, the treating physician should be aware of the possibility of illicit HA dermal filler injection as the culprit of DAH and myocardial infarction, even in a previously healthy patient.

\section{References}

1. Park TH, Seo SW, Kim JK, Chang CH. Clinical experience with hyaluronic acid-filler complications. J Plast Reconstr Aesthetic Surg 2011;64:892-6.

2. Han SW, Park MJ, Lee SH. Hyaluronic acid-induced diffuse alveolar hemorrhage: unknown complication induced by a well-known injectable agent. Ann Transl Med 2019;7:13-13.
3. Basora JF, Fernandez R, Gonzalez M, Adorno J. A case of diffuse alveolar hemorrhage associated with hyaluronic acid dermal fillers. Am J Case Rep 2014;15:199-202.

4. Park MS. Diffuse alveolar hemorrhage. Tuberc Respir Dis (Seoul) 2013;74:151-62.

5. Crea F, Libby P. Acute coronary syndromes: The way forward from mechanisms to precision treatment. Circulation 2017;136:1155-66.

6. Ben Gabr J, Kato H. A case of diffuse alveolar hemorrhage associated with high-titer of MPO-ANCA demonstrating cytoplasmic staining pattern. Case Rep Rheumatol 2019;2019:1-5.

7. Hammoudeh F, Perwaiz MK, Patolia S, et al. Diffuse alveolar haemorrhage with ANCA associated vasculitis - review of the literature. Br J Med Pract 2011;4:16-20.

8. West S, Arulkumaran N, Ind PW, Pusey CD. Diffuse alveolar haemorrhage in ANCA-associated vasculitis. Intern Med 2013;52:5-13.

9. Delorenzi C. Complications of injectable fillers, Part 2: Vascular complications. Aesthetic Surg J 2014;34:584-600.

10. Szczepanek K, Kieda C, Cichy J. Differential binding of hyaluronan on the surface of tissue-specific endothelial cell lines. Acta Biochim Pol 2008;55:35-42.

11. LeBoeuf RD, Gregg RR, Fuller GM, Weigel PH. Effects of hyaluronic acid and other glycosaminoglycans on fibrin polymer formation. Biochemistry 1987;26:6052-7.

12. Barbucci R, Benvenuti M, Casolaro M, et al. Sulfated hyaluronic acid as heparin-like material: physicochemical and biological characterization. J Mater Sci Mater Med 1994;5:830-3.

13. Esposito AJ, Bhatraju PK, Stapleton RD, et al. Hyaluronic acid is associated with organ dysfunction in acute respiratory distress syndrome. Crit Care 2017;21:304.

14. Herold S, Gabrielli NM, Vadász I. Novel concepts of acute lung injury and alveolar-capillary barrier dysfunction. Am J Physiol Lung Cell Mol Physiol 2013;305:L665-81. 